\title{
Towards a Generic Solution for Inspection of Industrial Sites
}

\section{Conference Paper}

Author(s):

Hutter, Marco (D); Diethelm, Remo; Bachmann, Samuel; Fankhauser, Peter; Gehring, Christian; Tsounis, Vassilios; Lauber, Andreas; Guenther, Fabian; Bjelonic, Marko; Isler, Linus; Kolvenbach, Hendrik; Meyer, Konrad; Hoepflinger, Markus

Publication date:

2017-09

Permanent link:

https://doi.org/10.3929/ethz-b-000183150

Rights / license:

In Copyright - Non-Commercial Use Permitted 


\title{
Towards a Generic Solution for Inspection of Industrial Sites
}

\author{
Marco Hutter, Remo Diethelm, Samuel Bachmann, Péter Fankhauser, Christian \\ Gehring, Vassilios Tsounis, Andreas Lauber, Fabian Guenther, Marko Bjelonic, \\ Linus Isler, Hendrik Kolvenbach, Konrad Meyer, Mark Hoepflinger
}

\begin{abstract}
Autonomous robotic inspection of industrial sites offers a huge potential with respect to increasing human safety and operational efficiency. The present paper provides an insight into the approach taken by team LIO during the ARGOS Challenge. In this international competition, the legged robot ANYmal was equipped with a sensor head to perform visual, acoustic, and thermal inspection on an oil and gas site. The robot was able to autonomously navigate on the outdoor industrial facilty using rotating line-LIDAR sensors for localization and terrain mapping. Thanks to the superior mobility of legged robots, ANYmal can omni-directionally move with statically and dynamically stable gaits while overcoming large obstacles and stairs. Moreover, the versatile machine can adapt its posture for inspection. The paper additionally provides insight into the methods applied for visual inspection of pressure gauges and concludes with some insight into the general learnings from the ARGOS Challenge.
\end{abstract}

\section{Introduction}

Recent advances in environment perception and robot control make autonomous mobile machines more and more applicable for inspection scenarios. Instead of expensive and inflexible instrumentation, mobile surface robots can carry sophisticated sensory equipment to any point of interest in order to conduct inspection or surveillance tasks. If such solutions are available, no humans need to be sent to working places that are dirty or dangerous, and even tedious and repetitive tasks can be conducted day and night with high precision.

Over the last years, there have been a number of initiatives from research and industry (e.g. EuRoC [16]) that try to bring such solutions from research laboratories

All authors are with the Robotic Systems Lab, ETH Zurich, Switzerland

e-mail: firstname.surnamedmavt.ethz.ch 
to real world sites. The ARGOS Challenge 1 , initiated by TOTAL SA, aims at the application of mobile robotic solutions for offshore oil and gas site inspection. According to [10], the organizers expect a major impact with respect to $i$ ) health, safety and environment (i.e. a reduction of risk to personnel, environment and installation as well as ii) operation (i.e. cost reduction, increase of efficiency and production). In the ARGOS Challenge, the robots must be able to autonomously navigate on the industrial site and inspect various objects such as pressure gauges, water level gauges, or valve handle positions. They need to analyze the sound of the running pumps in order to identify malfunctioning systems, detect alarm signals, find gas leaks as well as hot spots, and recognize changes that were made on the site (e.g. missing or moved objects). To make the scenario as realistic as possible, the applied robots must satisfy ATEX (explosion protection) and IP (ingress protection) standards. Moreover, during the missions that happen on multiple floors connected by steep stairs, the robots are facing different hurdles such as unexpected obstacles, heavy water falls, strong winds, or humans that are working on the site.

In contrast to specific robotic devices that are already commercially used in tanks, vessels, or pipes ${ }^{2}$ the ARGOS Challenge seeks for very generic robots that can oneto-one take over tasks performed by human specialists. In particular the requirements regarding mobility are demanding such that the few existing solutions like the wheel-based robots MIMROex [12] or SENSABOT[8] are not applicable. To address these issues, four of the five ARGOS Challenger teams selected in 2013 use tracked vehicles [10], while team LIO proposes an innovative solution based on a versatile legged robot. The remainder of this paper provides an insight into the realization of one of the most generic inspection robots.

\section{System Description}

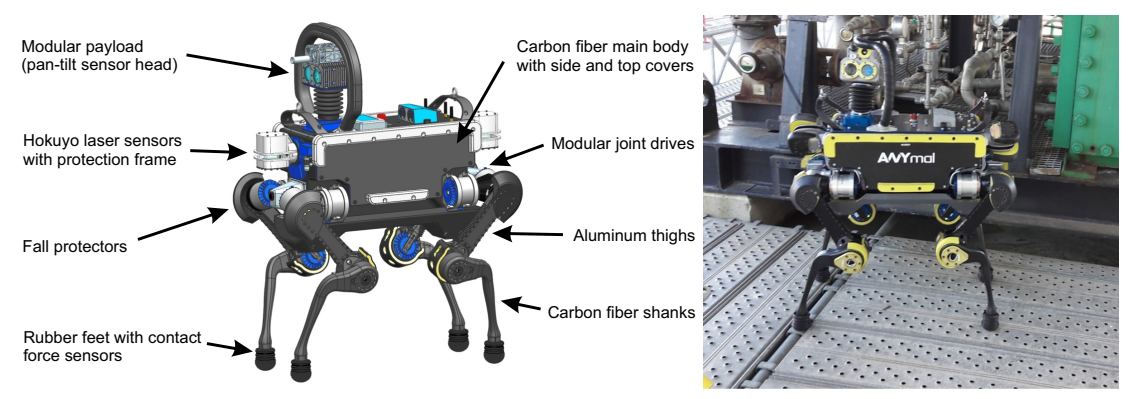

Fig. 1 ANYmal robot extended with a pan-tilt sensor head for inspection.

\footnotetext{
1 http://www.argos-challenge.com

${ }^{2}$ For example, see http://inspection-robotics.com
} 
Team LIO builds upon the modular and lightweight quadrupedal robot ANYmal [7] as transporter platform for inspection (Fig. 11). The legs of this versatile machine are driven by twelve equal series elastic actuator units [6] mounted at the joints. The kinematic structure of the robot is designed to achieve a large mobility allowing to overcome obstacles and stairs as well as for convenient transportation, compact storage, and simple deployment by a single operator. To keep the design as lightweight as possible, most of the structure is manufactured from carbon fibers. For fall protection, the robot features a rollover bar, a Kevlar belly plate, and shock absorbers. Moreover, force sensors in the feet provide haptic feedback of the environment, enabling safe locomotion even in case the robot is completely blind. ANYmal is designed in a hierarchical manner: On joint level, every actuator module is connected over a CAN bus and works independently. This allows component-level ingress and ATEX protection as well as fast and simple maintenance in case of hardware failure. On system level, computation is split among three independent computers that are connected through an internal network. The first computer (locomotion) hosts all real-time critical elements required for locomotion control and to interface the joint modules. The second computer (navigation) is responsible for environmental perception, localization, navigation, and mission execution, i.e. all software parts that are required to autonomously operate the robot. The third computer (inspection) runs all algorithms for inspection and detection.

For localization, navigation, and foothold planning, the machine is equipped with two rotating Hokuyo LIDAR sensors in the front and back, which provide detailed scans of the environment and the terrain. Additional wide-angle cameras in the front and back ensure an omni-directional view around the robot.

For inspection, we employ a pan-tilt head containing a high-quality zoom camera with high infrared (IR) sensitivity, a thermal camera, an ultrasonic and a regular microphone, a gas detection sensor, and LED illuminators. Since our robot can move its base in all directions, there is no need to employ the robot with an extension mechanism or arm.

The proposed system can operate fully autonomous with onboard batteries for more than 2.5 hours. To extend this lifetime, ANYmal can autonomously dock to recharge the battery and to pressurize the mainbody with Nitrogen for ATEX compliance [9].

\section{Autonomous Navigation on Industrial Sites}

The ability to autonomously move on industrial sites requires that the robot is able to precisely (and globally) localize, to map its environment, to plan a navigation path, as well as to detect and overcome obstacles. 


\subsection{Localization and Re-Localization}

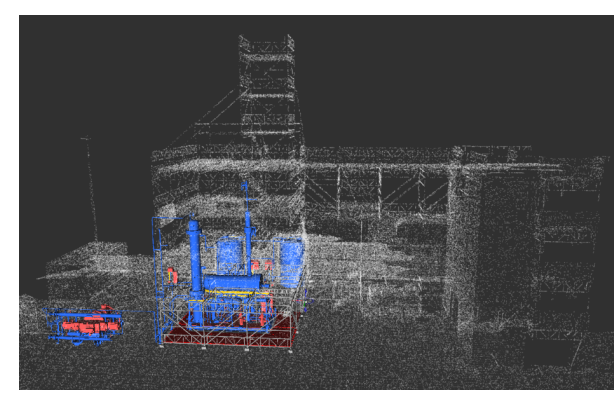

(a) Point Cloud

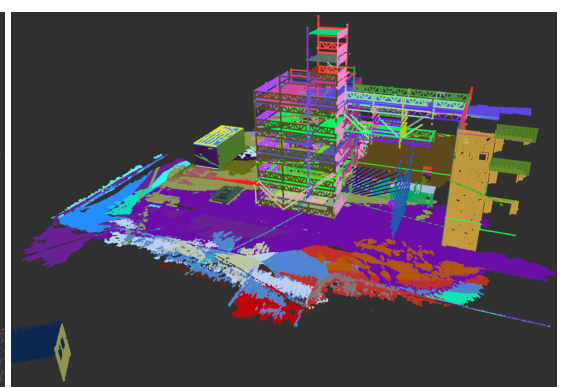

(b) Plane Segmentation

Fig. 2 a) The ICP algorithm matches every scan aquired by the LIDAR sensor during one half rotation with the known reference map by minimizing the distance between both point clouds. $b$ ) The point cloud of the site is segmented into planes for global localization.

To track its position and orientation, the robot scans its environment with two rotating line LIDAR sensors (Hokuyo UTM-30LX-EW) and matches the resulting 3D point cloud of to the reference map using the iterative closest point (ICP) algorithm [13]. The individual scans (half rotation of the laser, 20'000 points) are dewarped using the local state estimation from IMU and leg kinematics [1]. The ICP algorithm then searches for neighbor points between the two 3D point clouds of the single scan and the map (Fig. 2(a) and tries to minimize the sum of all their distances which takes about $0.4 \mathrm{~s}$. The estimated location is then fed back to the systems state estimator as an update measurement. The challenging part in this setup was that the duration of the ICP matching step is variable. To compensate for the potentially old position update, the pose was further propagated using the local state estimation [1].

To converge to the correct solution, ICP requires that the errors of the initial guess of the robots pose are less than $1.5 \mathrm{~m}$ in position and $30^{\circ}$ in orientation. In case of larger errors (i.e. at initialization or after loss of localization), the robot uses a plane matching algorithm. This algorithm searches for planes in the two 3D point clouds and groups them to all possible triples (see Fig. 2(b)]. A similarity analysis of a scan and a reference plane triple can be done quickly by comparing the interplanar angles. If the plane triples are similar, their relative transformation can be directly computed from the plane parameters. Using this transformation, the scanned point cloud is expressed in the reference frame and a plausibility analysis is done by computing the nearest neighbor ratio. If a certain threshold is met, the according relative transformation between the clouds is taken to derive the robots pose. This approach has proven to work very reliably during all missions and typically took between 20 and $100 \mathrm{~s}$ to find the right position. Wrong positives were not encountered in any of the missions. 


\subsection{Path Planning and Re-Planning}

The mission contains two consecutive states for path planning and path following. The path planning requires a start and a goal pose (whereby the start may be the robots current pose) and outputs a path. The path following takes this path as input and computes desired forward, sideways and rotational velocities with respect to the robots body frame for the locomotion controller.

For path planning, the robot features two complementary algorithms, namely the pose graph planner and the traversability planner.

The pose graph planner is used for global path planning, when the robot needs to navigate from one pose to another on the site. The pose graph (Fig. 3(a), which is created once for the entire site, is a representation of the accessible and safely traversable areas on the reference map. It consists of a tree of nodes with according connections (edges). Since the individual areas are mostly flat, the node is a degenerated pose containing position and yaw information. The type of motion that the robot is able to execute, i.e. the type of gait or climbing maneuver, is encoded in the edge. The pose graph planner computes a path by using an $\mathrm{A}^{*}$ algorithm on the node tree. This method has several advantages: The planning is very fast (due to the limited dimension of the problem) and the result is completely deterministic. Any two points on the site can be connected by the pose graph planner. If a point does not lie on the pose graph, the entry or exit nodes are determined by closest proximity evaluation. Thanks to the short planning time, the robot can continuously and in real-time re-plan its path independent on the event of blocked paths, changed missions, or emergency situations.

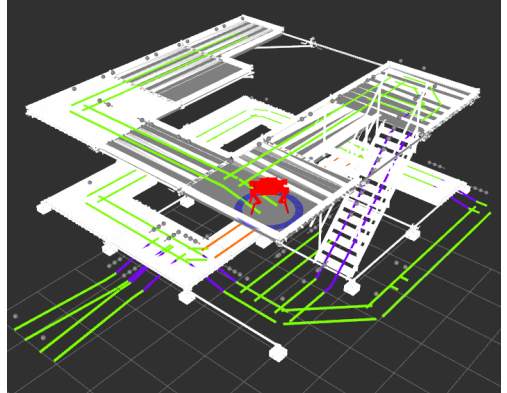

(a) Pose Graph

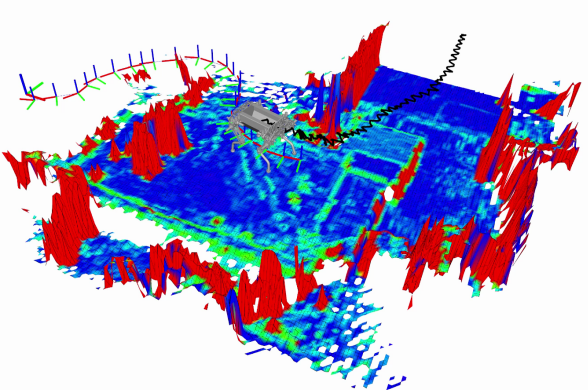

(b) Local Planner

Fig. 3 a) The pose graph consists of nodes (blue dots) and edges (green lines). b) While following the nominal trajectory, the traversability planner adapts the path around untraversable areas.

For local path planning, e.g. when an obstacle blocks the global path of the robot, the traversability planner [17 3 comes into play. It builds up a map containing the

\footnotetext{
${ }^{3}$ Traversability estimation online available: https://github.com/ethz-asl/traversability_estimation
} 
estimated traversability of the environment, which is computed by fusing various filters for slope, roughness, or step heights using the acquired LIDAR data (Fig. 3(b)). After planning the local path with a sampling based RRT* planner, the mission replaces the blocked segment of the global path with the alternative local path.

\subsection{Sense \& Move Strategies}

In cases where a checkpoint is hidden or badly visible from a selected viewpoint, e.g. due to reflections or bad angle of view, the robot visits an alternative position. To this end, a tool was developed to determine all possible inspection locations for every checkpoint, i.e. for every element that needs to be inspected. From the nominal position and orientation of the checkpoint given by the CAD model of the site, the possible inspection locations are determined. The likelihood of getting a good view on the checkpoint is evaluated as a function of the offset to the nominal robot posture and the angle of view offset (see Fig. 4p. The robot then starts with the optimal inspection point (green) and only moves to the others if the confidence for a correct inspection is below a defined treshhold.

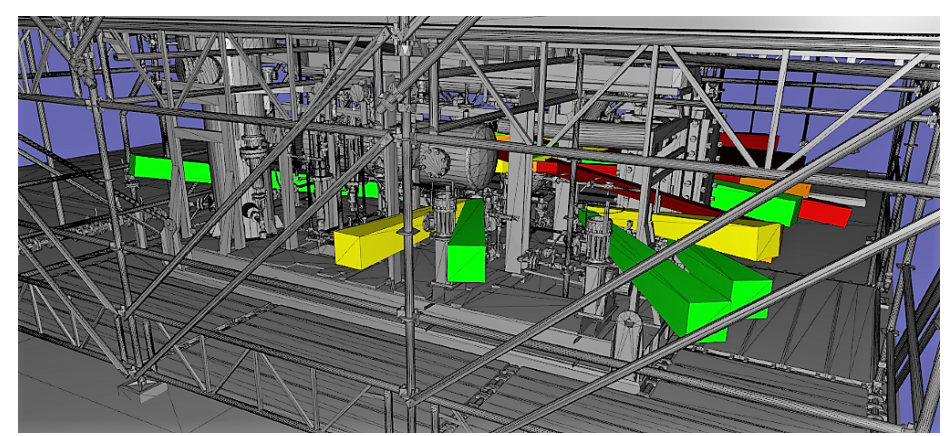

Fig. 4 Possible robot positions to read the checkpoint which are generated beforehand.

\subsection{Posture Adaptation}

At every inspection point, the robot adapts its posture within the kinematically feasible limits in order to get an optimal view of the checkpoint. As displayed in Fig.5. height and orientation of the body can be adjusted for every inspection situation. In fact, the robot can change height by about $0.5 \mathrm{~m}$, which allows for inspection without an additional arm. 

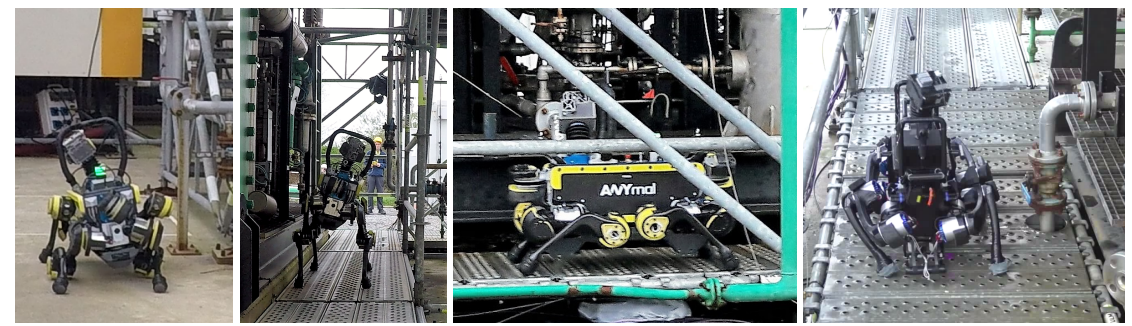

Fig. 5 ANYmal can adapt its posture to get an optimal view of the checkpoint.

\subsection{Stair Climbing}

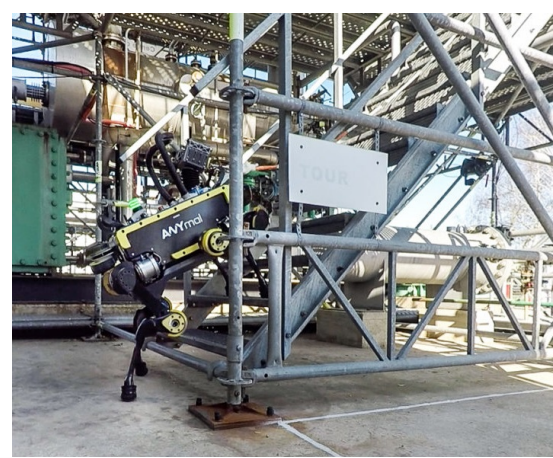

(a) Stair Walking

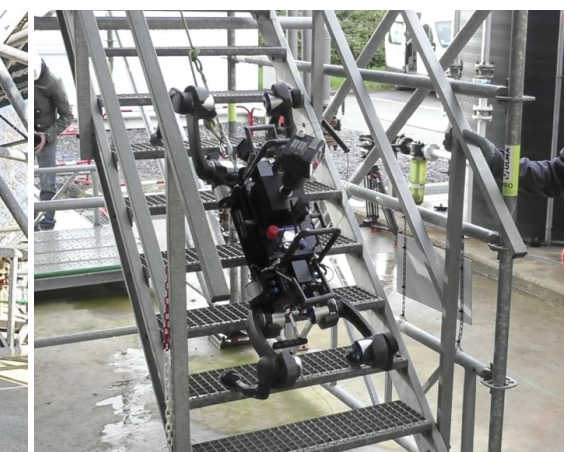

(b) Stair Crawling

Fig. 6 ANYmal uses the Free Gait motion control architecture [3] to overcome stairs by stepping regularly with one leg a time in a walking gait (a) or by using a turtle like crawling gait (b).

Ascending and descending stairs with ANYmal is achieved with two different strategies. In the general case, steps are negotiated by stepping regularly with one leg a time in a walking gait (Fig. 6(a)). The geometry of the stair such as the step rise and run is either taken from the CAD model or estimated online from the elevation map [4]. Based on this information, the foothold coordinates and whole-body motion is generated using the Free Gait motion control architecture 4 , where we use pose optimization and spline-based interpolation to automatically synthesize the required climbing motion [3]. In case of extreme conditions such as high inclinations $\left(>50^{\circ}\right)$, very slippery ground, or high wind speeds, ANYmal can negotiate the stairs in a turtle like crawling gait as demonstrated during the second competition. Here, the main body lies on the ground, the legs are moved to find the next stable contact holds, and the machine is subsequently pulled upwards (Fig 6(b)]. Thanks to ANY-

\footnotetext{
${ }^{4}$ Free Gait online available: https://github.com/leggedrobotics/free_gait
} 
mals large range of motion, the legs can be turned overhead to prevent collision with the ground or side rails.

\subsection{Obstacles}

\subsubsection{Obstacle Detection}

Building upon the onboard range measurement from the LIDAR sensors and the robot pose estimation, we create an elevation man ${ }^{5}$ which serves as a reference for obstacle detection [4]. The elevation map is a discrete 2.5D representation of the environment and it is based on the universal grid map library 6 , a mapping framework for mobile robotics [5]. An example of the visualization of a generated elevation map is shown in Fig. 7

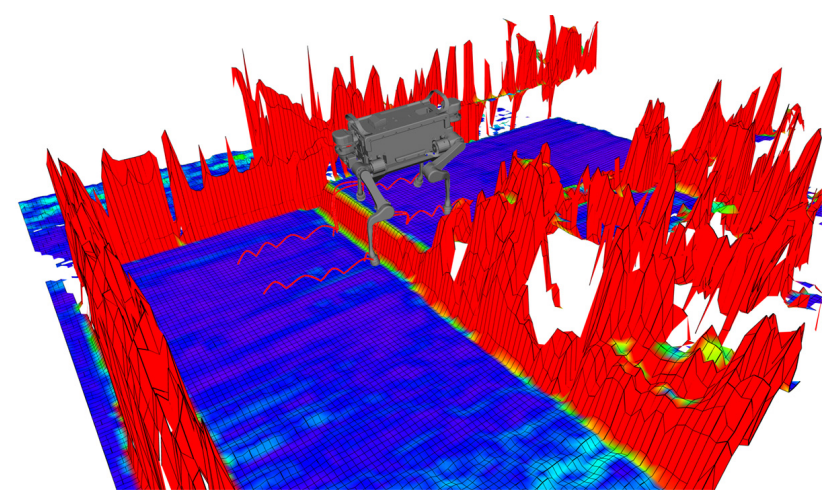

Fig. 7 Visualization of a generated elevation map during locomotion on site.

During operation, the robot updates the elevation map by periodically adding newly perceived point clouds. By comparing the reference elevation map of the site and the updated elevation map, changes in the environment and thus, obstacles are detected. In case of negative obstacles, this task becomes harder since they are defined as the absence of structure which can only be detected by ray-tracing. If a laser point behind an expected structural element is perceived, the robot assumes that the element has disappeared. To robustify the approach against outliers, we only classify clusters as an obstacle if at least several neighbor cells fall in the same region. Since laser data are sparse in far distance, only obstacles closer than $2.5 \mathrm{~m}$ are considered for path planning. In case obstacles fall within $2 \mathrm{~m}$ distance, the obstacle is additionally tested if it is a human or not.

\footnotetext{
${ }^{5}$ Elevation mapping online available under http://github.com/ethz-asl/elevation_mapping
}

${ }^{6}$ Grid map library online available under http://github.com/ethz-asl/grid_map 


\subsubsection{Human Detection}

The human detection is based on You Only Look Once (YOLO) [14] respectively the improved version YOLOv2 [15]. We adapted YOLOv2 to fit in our framework and use the small model of YOLOv2 with the training data from PASCAL VOC 2007 and 2012. Since the entire area around the robot must be covered, we use the front and back fisheye camera. As soon as the robot detects an obstacle inside the guarded space, the coordinates of the objects are transformed from Cartesian space into the image frames of the two cameras. Depending on the size of the obstacle, the image is cropped around the obstacles position to increase the robustness and speed of the human detection. Our version processes images on the central processing unit (CPU) and it only takes few seconds to detect objects.

\subsubsection{Obstacle Negotiation}

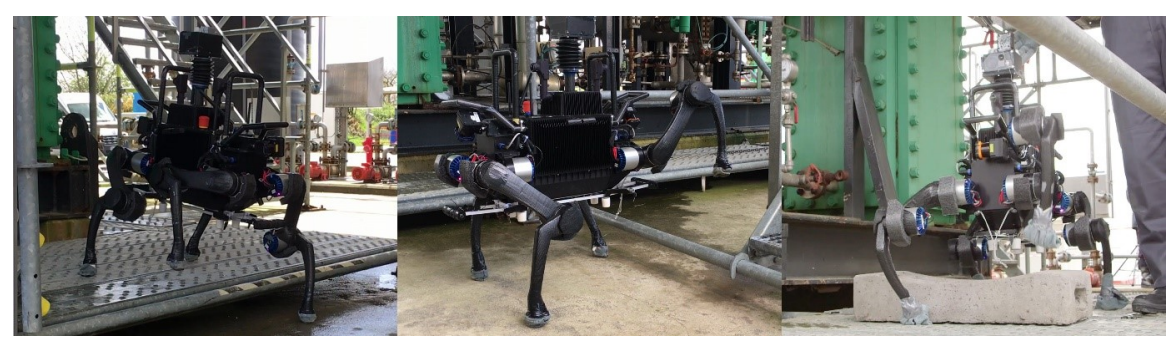

Fig. 8 ANYmal uses the Free Gait motion control architecture [3] to negotiate known (left and middle) and unknown (right) obstacles. For known obstacles we generated a database of parametrized motion definitions. Unknown obstacles are negotiated by selecting safe footholds from the environmental mapping and optimizing the body motion and posture.

Thanks to its legs and the high range of motion, ANYmal can flexibly climb over various known and unknown obstacles without creating contact with them. Small steps and obstacles (height $<20 \mathrm{~cm}$ ) are overcome by safely stepping on suitable footholds in a static walking gait (Fig. 8, left). Bigger obstacles and high steps (height $>20 \mathrm{~cm}$ ) are negotiated by rotating the legs in an outwards configuration (Fig. 8, center and right), which avoids any potential collision between the robot and the obstacles. With this, the robot can also climb on steps up to approximately $40 \mathrm{~cm}$ height. In case of known obstacles (Fig. 8, left and center), we defined the desired behavior (leg configuration and step positions) from a database of parameterized motion definitions. Based on our software architecture for motion generation [3], this allows for robust and repeatable obstacle negotiation. In case of unknown obstacles (Fig. 8, right), the elevation map created by the laser scanners is used to determine safe foothold locations. During the negotiation maneuver, the robot automatically adapts to the dimensions of the obstacle. 


\section{Inspection}

As outlined in the introduction, the robot is able to perform visual, thermal, and acoustic inspection. While a detailed description of all tools would go beyond the scope of this paper, we want to outline the specific approach and results for pressure gauge inspection.

\subsection{Camera Alignment, Tracking, Zooming}

In order to robustly point the camera at the checkpoint, the robot moves to a precomputed optimal posture at the inspection point and then determines online the required pan-tilt angles depending on the position of the robot and the checkpoint. After the approximate pan-tilt angles are set and the camera is aligned, the algorithm switches to a tracking mode. Tracking is required to zoom in without losing the checkpoint due to uncertainties in the robots position and position anomalies of the checkpoints. To track the checkpoint, we use a particle filter approach. The measurement updates for the particle filter come from analyzing the image with a histogram oriented gradients (HOG) [2] descriptor.

For a robust detector, a global HOG descriptor is trained with machine learning, namely the SVMlight library, using about 5000 positive and negative samples (Fig. 9). The positive samples are generated by rendering two different manometer models from different view points using blender and adding random background images from the ARGOS site. The negative samples consist only of random background images. Then, for each of those samples, a HOG descriptor vector is computed to train the global descriptor. The detector works with a sliding window approach and different image scales. To speed up the detection process, it is first searched only with the expected manometer size and stoped if at least two overlapping detections exist. If there were no detections or only one, different scales are applied to the image and the detector runs again.

This approach has proven to work very robustly since it does not matter if the pressure gauge has a different dial face. Once the checkpoint is tracked in the image, the camera zooms in until the checkpoint has the optimal size to read.

\subsection{Dewarping}

Usually it is not possible to face the camera exactly in front of the checkpoint as necessary for accurate reading. In order to dewarp the image by correcting the perspective and rotation of the checkpoint, two complementary approaches are implemented.

First, a given front image of the checkpoint is matched with a given example image by looking for scale-invariant feature transform (SIFT) features [11] that ap- 


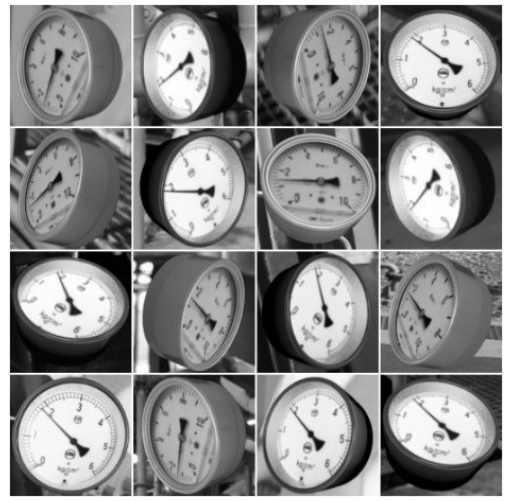

(a) Positive Samples

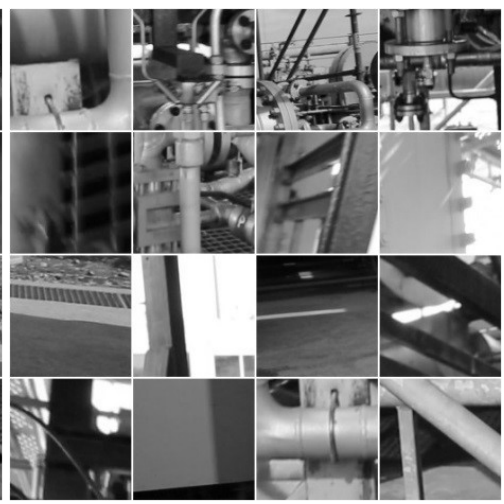

(b) Negative Samples

Fig. 9 Positive and negative examples for the machine learning algorithm.

pear in both images. From the matched SIFT features, the homography matrix is computed and used to dewarp the image (see Fig. 10p. This method requires an undistorted image of each pressure gauge type.

This SIFT-based approach may fail in situations where the image of the checkpoint differs too much from the given example image such that there are not enough features for image matching. This is typically the case if the angle of view is too large. To overcome this limitation, the algorithm can "manually" dewarp the taken image of the checkpoint with the knowledge of both the cameras orientation and the checkpoints nominal orientation. While this approach works well as long as the checkpoint has the nominal orientation, it is more likely to fail than the SIFT method if the checkpoint does not exhibit its nominal orientation.

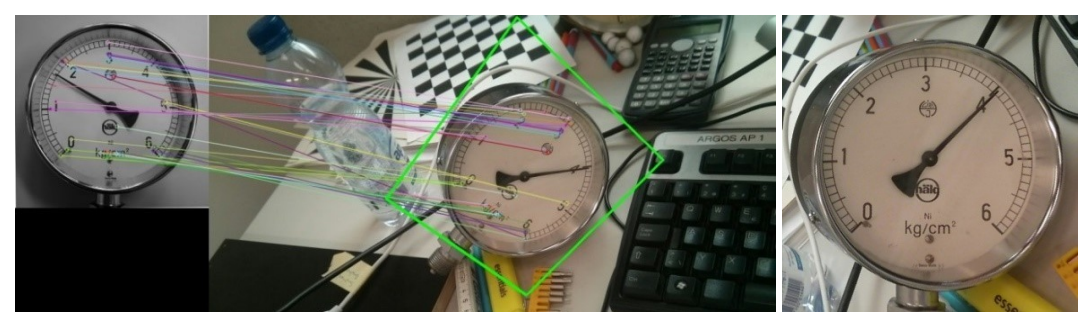

Fig. 10 The SIFT features are generated by comparing a given example image (left) with the image of the checkpoint (middle). The visualized homography matrix shows the connection lines between the matched features of both images. This homography matrix is used to generated the dewarped image (right). 


\subsection{Reading}

For an accurate reading of the pressure gauge it is important to identify the center of the pressure gauge. As illustrated in Fig. 11, a Hough-based circle detection is first applied to estimate the gauge frame. To limit the search area, the knowledge of the camera tracking is used as it provides an approximate position within the image. Once the circles are estimated, the mean is taken to provide the gauge frame and center of the gauge reading. In a second step, a Hough-based line detection is used to find the pointer of the pressure gauge. The algorithm uses the center to filter the lines and to discard the lines that do not belong to the pointer. The mean of the resulting lines gives the estimated pointer. Finally, the actual pressure value is computed from the known scale of the pressure gauge, the center, and the line angle.

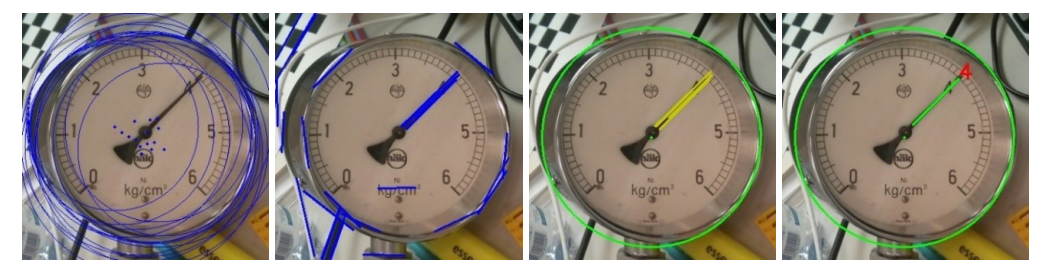

Fig. 11 Pressure gauge reading process (from left to right): 1) multiple circle detections, 2) line segment extractions, 3 ) resulting mean circle with estimated manometer center (green point) and filtered lines (yellow), 4) mean pointer line (green) and resulting read value in red.

\subsection{Evaluation}

To verify the robustness of the pressure gauge reading algorithm, a series of measurements from different viewpoints and with different pointer positions was conducted (Fig. 12).

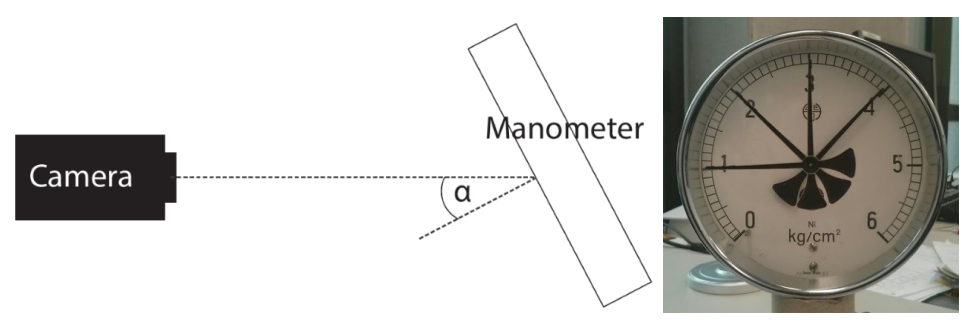

Fig. 12 Experimental setup to identify the maximal possible angle of view. 


\begin{tabular}{lccccccccc}
\hline Defined angle $\alpha^{\prime}$ & $0^{\circ}$ & $10^{\circ}$ & $20^{\circ}$ & \multicolumn{2}{c}{$40^{\circ}$} & \multicolumn{2}{c}{$45^{\circ}$} & $50^{\circ}$ \\
Actual angle $\alpha$ & $0^{\circ}$ & $10^{\circ}$ & $20^{\circ}$ & $40^{\circ}$ & $50^{\circ}$ & $45^{\circ}$ & $55^{\circ}$ & $50^{\circ}$ \\
\hline Pointer position & \multicolumn{3}{c}{ Measurements } & & & & & & \\
$1.00 \mathrm{~kg} / \mathrm{cm}^{2}\left(\sim 90^{\circ}\right)$ & 0.96 & 0.96 & 0.96 & 0.96 & 0.94 & 0.96 & 0.92 & 0.96 \\
$1.99 \mathrm{~kg} / \mathrm{cm}^{2}\left(\sim 135^{\circ}\right)$ & 1.97 & 1.97 & 1.99 & 1.99 & $\underline{2.15}$ & 2.00 & $\underline{2.18}$ & 2.03 \\
$2.98 \mathrm{~kg} / \mathrm{cm}^{2}\left(\sim 180^{\circ}\right)$ & 2.92 & 2.96 & 2.97 & 2.97 & 3.01 & 3.00 & 3.02 & 3.02 \\
$3.98 \mathrm{~kg} / \mathrm{cm}^{2}\left(\sim 225^{\circ}\right)$ & 3.98 & 3.98 & 3.97 & 3.98 & $\underline{3.88}$ & 3.98 & $\underline{3.86}$ & 3.96 \\
\hline SIFT rate & $100 \%$ & $100 \%$ & $100 \%$ & $100 \%$ & $0 \%$ & $91 \%$ & $0 \%$ & $12 \%$ \\
\hline
\end{tabular}

Table 1 Measurements with different angles and pointer positions. 50 measurements per angle and pointer position were obtained.

The results displayed in Tab. 1 proof that the overall robustness of the proposed approach is high. Ignoring the cases where $\alpha \neq \alpha^{\prime}$ gives an average absolute deviation of $0.013 \mathrm{~kg} / \mathrm{cm}^{2}$ respectively $0.58^{\circ}$ with an allowed maximal deviation of $0.12 \mathrm{~kg} / \mathrm{cm}^{2}$ or $5.36^{\circ}$. Even the manual dewarping at $\alpha \neq \alpha^{\prime}=50^{\circ}$ provides accurate measurements (only $12 \%$ of the images were dewarped by the SIFT algorithm). Overall, manometers can be read within the required accuracy from angles of view between $-50^{\circ}$ and $50^{\circ}$. The experience during the actual inspection missions supports these results as outstanding inspection accuracy was achieved in all missions.

\section{Conclusion}

The present paper illustrates the worldwide first attempt of using a versatile legged machine for autonomous inspection on industrial sites. In three consecutive competition on a testing site in Pau, France, team LIO was able to demonstrate the high potential of the proposed solution. For illustration, we collected a video summary of Challenge $2^{7}$ and Challenge $3^{8}$

While all individual tasks regarding system mobility, navigation, and inspection could be entirely fulfilled, the high system complexity entails several potential sources for failure. Firstly, the fact that neither stopping (blocking) nor disabling the joint motors leads to an immediate stop of a legged robot complicates safety consideration. As a result, we encountered during the three competitions several (uncontrolled) falls which the robot all survived. In the future, it is required to further extend the work on smart emergency behaviors such that legged robots can safely operate even in case of critical software or hardware failure. Secondly, the realistic missions unveiled that robust, reliable, and fast terrain perception under harsh conditions is still challenging. When the robot was moving fast, it was difficult to create terrain maps without any false positive obstacles due to the distorted scans from the motion, reflections on metal and wet surfaces, and rain drops on the laser. Thirdly,

7 https://youtu.be/SR5OJ-vklIs

8 https://youtu.be/2RQDp0Q2vSo 
despite the superiority in mobility compared to tracked or wheeled vehicles, there is still a lot of potential for improvement of the locomotion skills of legged robots. Once these deficiencies are overcome, we are convinced that legged robots such as ANYmal can find their way into applications like industrial inspection.

\section{References}

1. M. Bloesch, M. Hutter, M. Hoepflinger, S. Leutenegger, C. Gehring, C. D. Remy, and R. Siegwart. State Estimation for Legged Robots - Consistent Fusion of Leg Kinematics and IMU. In Robotics Science and Systems (RSS), pages 17-24, 2012.

2. N. Dalal and B. Triggs. Histograms of Oriented Gradients for Human Detection. In IEEE Computer Society Conf. on Computer Vision and Pattern Recognition, pages 886-893, 2005.

3. P. Fankhauser, C. D. Bellicoso, C. Gehring, R. Dube, A. Gawel, and M. Hutter. Free Gait An architecture for the versatile control of legged robots. In IEEE-RAS 16th International Conference on Humanoid Robots (Humanoids), pages 1052-1058. IEEE, nov 2016.

4. P. Fankhauser, M. Bloesch, C. Gehring, M. Hutter, and R. Siegwart. Robot-Centric Elevation Mapping with Uncertainty Estimates. In International Conference on Climbing and Walking Robots (CLAWAR), pages 433-440, 2014.

5. P. Fankhauser and M. Hutter. A Universal Grid Map Library: Implementation and Use Case for Rough Terrain Navigation. In Robot Operating System (ROS) - The Complete Reference, pages 99-120. Springer, 2016.

6. M. Hutter, K. Bodie, A. Lauber, and J. Hwangbo. EP16181251 - Joint unit, joint system, robot for manipulation and/or transportation, robotic exoskeleton system and method for manipulation and/or transportation, 2016.

7. M. Hutter, C. Gehring, D. Jud, A. Lauber, C. D. Bellicoso, V. Tsounis, J. Hwangbo, K. Bodie, P. Fankhauser, M. Bloesch, R. Diethelm, S. Bachmann, A. Melzer, and M. Hoepflinger. ANYmal - a highly mobile and dynamic quadrupedal robot. In IEEE/RSJ International Conference on Intelligent Robots and Systems (IROS), pages 38-44. IEEE, oct 2016.

8. JPT Staff. Sensabot: A Safe and Cost-Effective Inspection Solution. Journal of Petroleum Technology, 64(10):32-34, 2012.

9. H. Kolvenbach and M. Hutter. Life Extension: An Autonomous Docking Station for Recharging Quadrupedal Robots. In (submitted to) Field and Service Robots (FSR), 2017.

10. K. Kydd, S. Macrez, and P. Pourcel. Autonomous Robot for Gas and Oil Sites. In SPE Offshore Europe Conference and Exhibition. Society of Petroleum Engineers, sep 2015.

11. D. Lowe. Object recognition from local scale-invariant features. In IEEE International Conference on Computer Vision, pages 1150-1157. IEEE, 1999.

12. K. Pfeiffer, M. Bengel, and A. Bubeck. Offshore robotics - Survey, implementation, outlook. In IEEE/RSJ International Conference on Intelligent Robots and Systems, pages 241-246, 2011.

13. F. Pomerleau. Applied registration for robotics. PhD thesis, ETH, 2013.

14. J. Redmon, S. Divvala, R. Girshick, and A. Farhadi. You Only Look Once: Unified, Real-Time Object Detection. In IEEE Conference on Computer Vision and Pattern Recognition, pages 779-788, 2016.

15. J. Redmon and A. Farhadi. YOLO9000: Better, Faster, Stronger. arXiv preprint arXiv:1612.08242, dec 2016.

16. B. Siciliano, F. Caccavale, E. Zwicker, M. Achtelik, N. Mansard, C. Borst, M. Achtelik, N. O. Jepsen, R. Awad, and R. Bischoff. EuRoC - The Challenge Initiative for European Robotics. In International Symposium on Robotics; Proceedings of ISR/Robotik, 2014.

17. M. Wermelinger, P. Fankhauser, R. Diethelm, P. Krusi, R. Siegwart, and M. Hutter. Navigation planning for legged robots in challenging terrain. In IEEE/RSJ International Conference on Intelligent Robots and Systems (IROS), pages 1184-1189. IEEE, oct 2016. 